\title{
Beam Lifetime Dependence on the Beam-Gas Interactions in RHIC*
}

\author{
D. Trbojevic, H. C. Hsueh, W. MacKay, A. Drees, and R. Fliller * , BNL, UPTON, N.Y. 11973, USA
}

\section{Abstract}

In the Relativistic Heavy ion Collider (RHIC) much larger background signals were occurring at BRAMS, one of the four experiments. This was especially pronounced at the time when vacuum conditions deteriorated due to the beam ionization profile monitor replacements. Recording the beam intensities during the store provided the beam lifetime. Predictions from the beam gas interactions to the above measured values are compared. The ionization gauges simultaneously recorded the vacuum pressure data.

\section{INTRODUCTION}

In the normal operating conditions the beam gas interaction should not be a dominant effect on beam lifetime in RHIC. Most of the RHIC beam pipe (80\%) is at $5 \mathrm{~K}$, while "warm"sections at $300 \mathrm{~K}$ make for about $20 \%$ of the circumference. In the RHIC "cold" part helium molecules are making $99 \%$ of the residual gas pressure composition. With beam pipe walls of the RHIC "cold" part at $5 \mathrm{~K}$, and with additional ion and special getter pumps provided for the helium molecules, the maximum gas pressure is less than $10^{-11}$ Torr. To achieve required operating pressure of $10^{-10}$ Torr in the warm regions, vacuum pipes need to be backed out. As will be shown later a projected beam lifetime just due to beam-gas effect was more than $\tau \geq 240$ hours. A gas composition at room temperature in the "warm" part is made of mostly hydrogen molecules $(\sim 90 \%)$ and the rest of the spectrum are methane $\left(\mathrm{CH}_{4}\right)$ $\sim 5 \%$, and carbon dioxide $\sim 5 \%\left(\mathrm{CO}_{2}\right)$ molecules. The water molecules are removed by the bake-out. During the commissioning some of the warm sections were not completely baked out. After the regular stores of the two gold beams with luminosities at detectors were established, in few occasions due to necessary instrumentation repairs, the vacuum system had to be disturbed. The room temperature beam pipe had to be open. At times when RHIC operation restarted immediately after the repairs and vacuum breaks, the background noise around this area at the "BRAMS" detector became significantly higher. The beam life time of one of the beams through the area of repair became much shorter as shown in Fig. 1 and in the opposite ring as shown in Fig. 2.

\subsection{Basic parameters}

A standard RHIC operation during the run in the summer of 2000 was with 56 bunches per ring, with an average gold beam of $2 \cdot 10^{8}$ ions per bunch. The revolution frequency is

* Work performed under the auspices of the U.S. Department of Energy

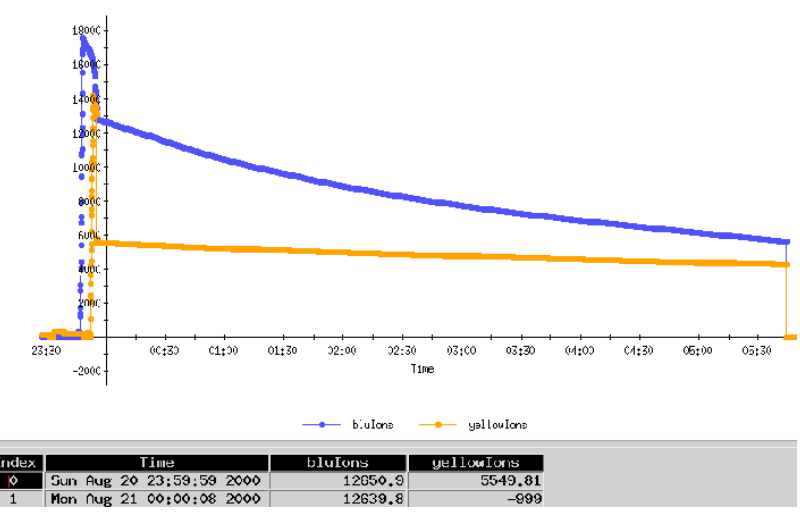

Figure 1: Life times of both "blue" and the "yellow" beams shown by the beam DCCT current monitors after the blue ring repair in the warm region.

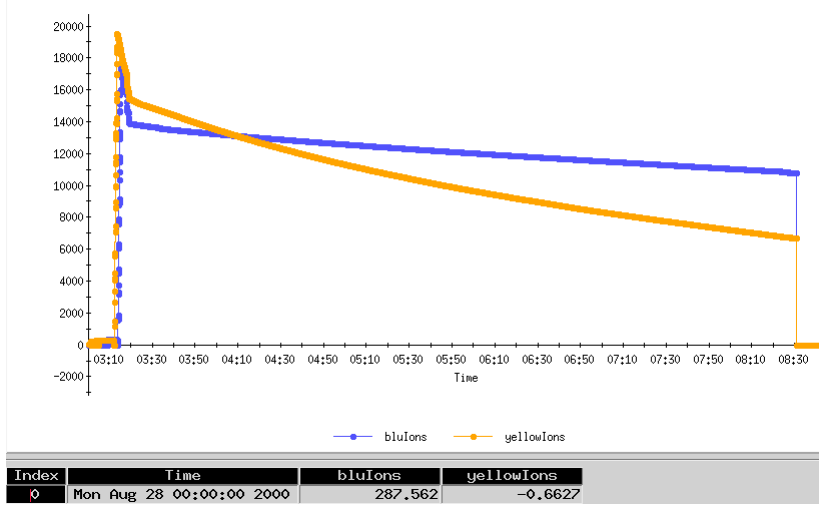

Figure 2: Life times of both "blue" and the "yellow" beams shown by the DCCT beam current monitors after the yellow ring repair in the warm region.

$78.189 \mathrm{kHz}$ (or the revolution period of $\mathrm{T}=12.789 \mu \mathrm{s}$ ). The average gold ion currents per ring are:

$$
I_{\text {Gold }} \cong \frac{56 \cdot 2 \cdot 10^{8} \cdot 79^{+} \cdot 1.602 \cdot 10^{-19}}{12.789 \cdot 10^{-6}} \cong 11 \mathrm{~mA}
$$

The beam lifetime is influenced by the dominant central nuclear collisions. The cross sections for the beamgas electron radiative captures are an order of magnitude smaller. The capture cross sections include the radiative and non-radiative capture, and the capture from the electron-positron pair creation from the "vacuum capture". The beam lifetime is presented as an exponential decay [1], with the loss rate of the beam particles shown in Eq. 1:

$$
\frac{1}{\tau}=\frac{1}{N} \frac{d N}{d t}=n l f\left(\sigma_{N}+\sigma_{C}\right)
$$


where $f$ is the revolution frequency, $l$ is the path of the projectiles (the circumference of RHIC is equal to $C=3833.845 \mathrm{~m}$ ), and $n$ is the density of molecules within the beam pipe. The cross sections [2] for the radiative and non-radiative electron captures, $\sigma_{R C}=a Z_{P}^{5} Z_{T} / \gamma$ and $\sigma_{N R C}=b Z_{P}^{5} Z_{T}^{5} / \gamma$, respectively, as well as the capture cross section from the beam gas electron-positron creation $\sigma=d Z_{P}^{5} Z_{T}^{2} \ln \left(\gamma / \gamma_{o}\right)$, are neglected in this report. The parameters in the cross sections are the charge of the projectiles-P and target-T, $Z_{P}$ and $Z_{T}$, respectively, the relativistic factor $\gamma$ and parameters $\gamma_{o}, a, b$, and $d$ with values provided in ref.[2]. The cross section of the nuclear scattering $\sigma_{N}$ in collisions between the projectiles-P ions and the residual gas target-T nuclei, treated as "billiard balls" is given by Eq. 2:

$$
\sigma_{N} \simeq \pi R_{N}^{2}, \text { with } R_{N} \simeq r_{o} A^{\frac{1}{3}}, \text { where } r_{o}=1.2 \mathrm{fm},
$$

For warm vacuum sections the cross sections between the gold ions and the gas molecules is presented as a weighted sum of the three cross sections by Eq. 3:

$$
\sigma_{N_{\text {tot }}}=0.9 \sigma_{H_{2}}+0.05 \sigma_{C_{H_{4}}}+0.05 \sigma_{C O}
$$

where $\sigma_{A u, H_{2}}=2.267 \cdot 10^{-24} \mathrm{~cm}^{2}, \sigma_{A u, C H_{4}}=4.622$. $10^{-24} \mathrm{~cm}^{2}$, and $\sigma_{A u, C O}=5.110 \cdot 10^{-24} \mathrm{~cm}^{2}$. The cross section for the central nuclear collisions between the gold beam and the residual gas in the warm part of RHIC is estimated to be $\sigma_{N} \simeq 2.53 \cdot 10^{-24} \mathrm{~cm}^{2}$, while for the cold section the cross section is estimated to be $\sigma_{N} \simeq$ $2.48 \cdot 10^{-24} \mathrm{~cm}^{2}$. The density of molecules within the beam pipe $n$ is given by known Eq. 4:

$$
n=\frac{p}{k T}=9.656 \cdot 10^{18} \frac{p(\text { Torr })}{T} \text {, or } p V=\nu_{\text {mol }} R T \text {, }
$$

where $p$ is the residual gas pressure in the beam pipe, $\nu_{\text {mol }}$ is the number of moles, $R$ is the molar gas constant $R \equiv$ $k N_{A}=8.314510 \cdot 10^{7}(\mathrm{erg} / \mathrm{Kmol})$, where $N_{A}$ is the Avogadro's number $N_{A}=6.0221367 \cdot 10^{23}($ molecules $/ \mathrm{mol})$ the Boltzmann constant $\mathrm{k}=1.380662 \cdot 10^{-16}$ (erg/(molecule $\mathrm{K})$ ), the gas density $n$ (molecules $/ \mathrm{cm}^{3}$ ), and the $\mathrm{T}(\mathrm{K})$ is the gas temperature. With a pressure of $p=1 \cdot 10^{-9}$ (Torr) in the warm part of RHIC $l=0.2 \cdot C$, the lifetime of the gold ions is:

$$
\tau=\frac{1}{p(\text { Torr })} \frac{1}{0.2} \cdot 1.13710^{-7} \text { hours } \simeq 570 \text { (hours). }
$$

, while the lifetime of the gold ions in the "cold part" of RHIC where $l=0.8 \cdot C$, with the temperature of the walls estimated to be $5 \mathrm{~K}$, with a pressure of $p=1 \cdot 10^{-11}$ Torr, is:

$$
\left.\tau=\frac{1}{p(\text { Torr })} \frac{1}{0.8} \cdot 1.935 \cdot 10^{-9} \text { (hours }\right) \simeq 240 \text { (hours). }
$$

\section{VACUUM MEASUREMENTS}

During both presented beam stores in Fig. 1 which happened on August 22, 2000 and Fig. 2 on August 28, 2000, when experiments were recording collisions, results from the vacuum measurements in the cold part of the RHIC vacuum system showed pressures of less than $p<10^{-11}$ Torr. The vacuum pressures measurements in the area where the repair work happened are shown in Fig. 3 and in the opposite ring as shown in Fig. 4. It is interesting to note, that even at the warm regions where the average vacuum pressure was less than $1 \cdot 10^{-9}$ Torr a very distinguished bumps in the vacuum corresponded to the beam stores. A time of rise of the vacuum pressure corresponded directly to the time of the beam injection, and the fall of the pressure signal corresponded to the beam abort. In the warm

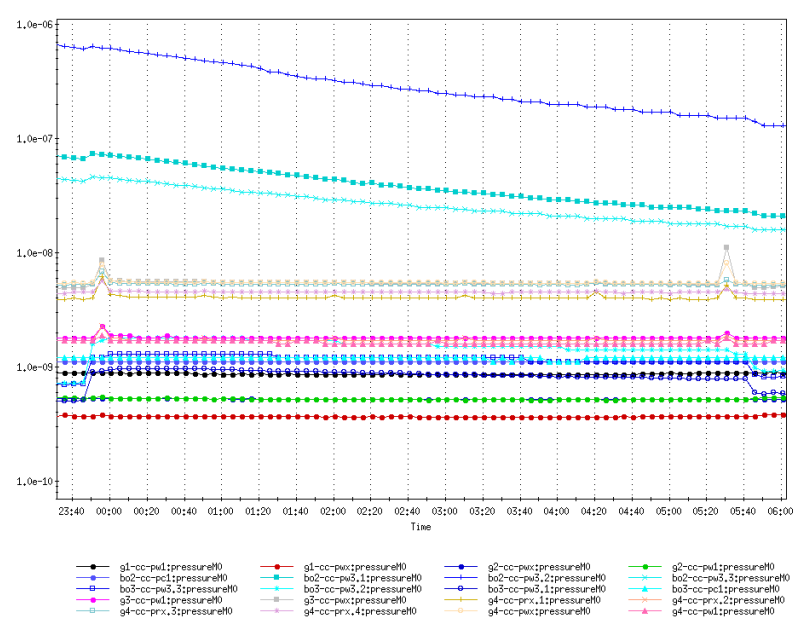

Figure 3: Vacuum pressures in the region where a repair was previously done on August 21, 2000.

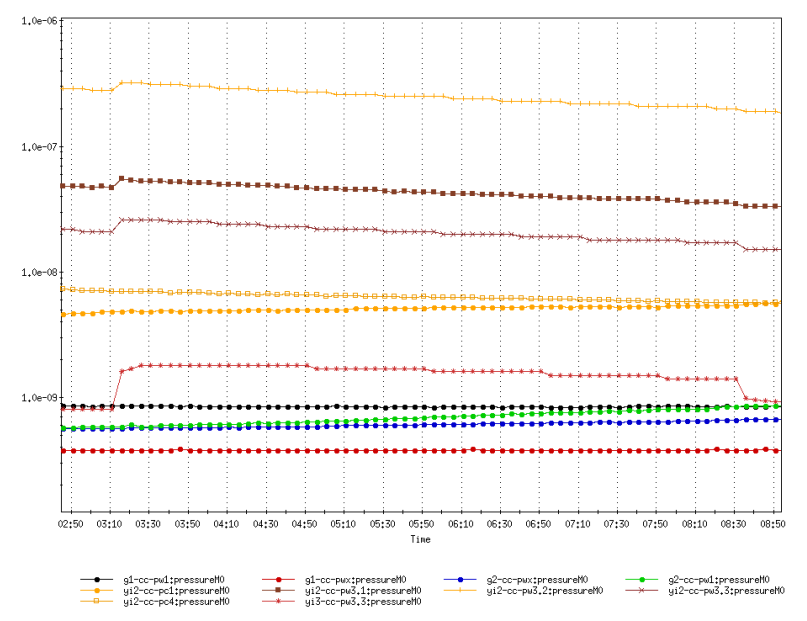

Figure 4: Vacuum pressures in the region where a repair was previously done on August 27, 2000.

regions average values of the pressures, measured on $\mathrm{Au}-$ gust 22, 2000 are shown in Table 1. According to the Eq. 1 the inverse value of the beam life time is equal to the sum of all contributions from the beam-gas interactions around the ring. The last two rows of both tables show both estimated life time from the pressure data around the ring (the 
cold part of both rings is taken with the life time of $\tau \sim 240$ hours, compared to the measured beam life time obtained from Fig. 1 and Fig. 2. In the warm regions average values

Table 1: Pressures in the warm sections August 22

\begin{tabular}{|l|c|c|}
\hline Sectors & Blue(Aug.22)Torr & Yellow(Aug.22)Torr \\
\hline $1 \& 12$ & $2.8 \cdot 10^{-8}$ & $3.8 \cdot 10^{-9}$ \\
$2 \& 3$ & $3.1 \cdot 10^{-7}$ & $1.0 \cdot 10^{-8}$ \\
$4 \& 5$ & $1.3 \cdot 10^{-8}$ & $1.1 \cdot 10^{-8}$ \\
$6 \& 7$ & $4.1 \cdot 10^{-9}$ & $6.2 \cdot 10^{-9}$ \\
$8 \& 9$ & $2.1 \cdot 10^{-9}$ & $1.1 \cdot 10^{-8}$ \\
$10 \& 11$ & $1.2 \cdot 10^{-9}$ & $1.2 \cdot 10^{-9}$ \\
\hline$\tau_{\text {total }}$ & 8.84 hours & 58.22 hours \\
\hline$\tau_{\text {measur. }}$ & 8.56 hours & 24.37 hours \\
\hline
\end{tabular}

of the pressures, measured on August 28, 2000 are shown in Table 2:

Table 2: Pressures in the warm sections August 28

\begin{tabular}{|l|c|c|}
\hline Sectors & Blue (Aug.28)Torr & Yellow (Aug.28)Torr \\
\hline $1 \& 12$ & $8.1 \cdot 10^{-9}$ & $4.5 \cdot 10^{-9}$ \\
$2 \& 3$ & $1.0 \cdot 10^{-8}$ & $3.8 \cdot 10^{-7}$ \\
$4 \& 5$ & $1.1 \cdot 10^{-8}$ & $2.3 \cdot 10^{-8}$ \\
$6 \& 7$ & $4.1 \cdot 10^{-9}$ & $5.0 \cdot 10^{-9}$ \\
$8 \& 9$ & $2.5 \cdot 10^{-9}$ & $3.4 \cdot 10^{-9}$ \\
$10 \& 11$ & $3.0 \cdot 10^{-9}$ & $2.5 \cdot 10^{-9}$ \\
\hline$\tau_{\text {total }}$ & 64 hours & 12.36 hours \\
\hline$\tau_{\text {measur. }}$ & 22.6 hours & 6.37 hours \\
\hline
\end{tabular}

\section{TRANSVERSE EMITTANCE GROWTH DUE TO BEAM GAS INTERACTIONS}

The beam transverse emittance growth in RHIC is expected to be mostly due to intra-beam scattering of the fully stripped gold ions. The Rutherford Coulomb scattering cross section for an incident particle with a speed " $\mathrm{v}$ " and charge " $e$ " approaching a target nucleus of charge $Z \cdot e$ with an impact parameter "b" is [3] provided by the very well known Rutherford Coulomb scattering Equation 5:

$$
\frac{d \sigma}{d \Omega}=\frac{b}{\theta} \frac{d b}{d \theta}=4\left(\frac{Z e^{2}}{4 \pi \epsilon_{o} p v}\right)^{2} \frac{1}{\theta^{4}} .
$$

Other details of the beam growth calculations are presented elsewhere [4]. A dependence of the beam growth due to multiple Coulomb scattering with the residual gas molecules in the normal vacuum conditions is expected to be very small. The emittance growth per hour of the gold ion beam in the warm sections of RHIC ( $\mathrm{T}=300 \mathrm{~K})$, with the previously estimated value of the average beta function of $\beta_{\text {avr }} \simeq 30 \mathrm{~m}$, is calculated [4] as Eq. 6:

$$
\frac{d \epsilon_{N}}{d t} \simeq 4.57 \cdot 10^{6} \cdot 0.2 \frac{\beta \cdot \mathrm{p}(\text { Torr })}{\gamma}(\mathrm{mmmrad} / \text { hour })
$$

where in the RHIC run2000 a value of the relativistic factor during the stored beams was $\gamma=75.18$. The normalized emittance growth in the warm part of the RHIC (20\%) at the estimated pressure of $\mathrm{p}=1 \cdot 10^{-9}$ Torr and is calculated [4] as:

$$
\frac{d \epsilon_{N}}{d t} \simeq 3.65 \cdot 10^{-4}(\mathrm{~mm} \mathrm{mrad} / \text { hour }) .
$$

During the period of repairs, previously mentioned, the vacuum conditions did not correspond to the design values. Applying the equation Eq. 6 in both of conditions above on August 22, when the average pressure in the blue ring was $p_{\text {avrg }}=5.97 \cdot 10^{-8}$, and on August 28, 2000 when the average pressure in the yellow ring was $p_{\text {avrg }}=6.97 \cdot 10^{-8}$ Torr. The beam growths at these two conditions:

$$
\begin{aligned}
& \frac{d \epsilon_{N}}{d t}\left(\text { Aug.22) } \simeq 2.18 \cdot 10^{-2}(\mathrm{~mm} \mathrm{mrad} / \text { hour }),\right. \\
& \frac{d \epsilon_{N}}{d t}\left(\text { Aug.28) } \simeq 2.54 \cdot 10^{-2}(\mathrm{~mm} \mathrm{mrad} / \text { hour }) .\right.
\end{aligned}
$$

\section{SUMMARY}

A comparison between the calculated beam life time with respect to the measured one had shown relatively good agreement, at unusual conditions in RHIC which occurred during periods when necessary repairs were performed. The expected beam growth of the fully stripped gold ions due to the intra-beam scattering in the previous run 2000 was not conclusive due to often disturbed conditions in the warm part of the RHIC vacuum. This report showed that at few periods of operation the transverse beam emittance growth may had been dominated by the beam-gas interactions.

\section{REFERENCES}

[1] D. Trines, "The Hera Cold Bore Vacuum System", DESY HERA 85-22, October 1985.

[2] M.J. Rhoades-Brown and M. Harrison, "Vacuum Requirements for RHIC”, BNL-47070, AD/RHIC-106, Informal Report, December 1991.

[3] M. Syphers and D. Edwards, "An Introduction to the Physics of High Energy Accelerators", John Wiley \& Sons, Inc., 1993, pp. 250.

[4] D. Trbojevic, "The Beam Life Time and Emittance Growth in RHIC under Normal Operating Conditions and with a Hydrogen Gas Jet", Internal Report, Brookhaven National Laboratory, Report-No: RHIC/AP/136, October 1997, http : //www.cadops.bnl.gov/AP/apnotes/rapindex.html. 